\title{
Einführung eines Peer Review by request-Verfahrens
}

Von Klaus-Dieter Altmeppen und Alexander Filipović

$\mathrm{E}$ $\mathrm{s}$ ist fast wie bei parship, nur gesellschaftlich folgenreicher: „Alle 20 Sekunden erscheinen ein Forschungsaufsatz und jeden Tag mehr als 240 Bücher, hat die Frankfurter Allgemeine Zeitung 2013 errechnet" (Dannenberg 2017, S. 23). Durch diese Prozesse sind häufig aber nur die Gutachter_innen im Besitz exklusiven Wissens, denn „nur die Hälfte der in zitierten Zeitschriften erschienenen Artikel würden von anderen als den Autoren und den Gutachtern gelesen, und 9o Prozent der Artikel niemals zitiert, zählte der Wirtschaftswissenschaftler Prof. Dr. Alfred Kieser 2016" (ebd.; s. auch Frey/Osterloh 2016, S. 134 f.). Das allein schon sollte allen zu denken geben, die - als Betreuer_innen oder als Promovend_innen - kumulierte Promotionen fordern und fördern (vgl. z. B. Guenther 2017, S. 5).

Angesichts dieser Zahlen erscheint die bisherige Entscheidung von Herausgebern und Redaktion der Communicatio Socialis, kein Reviewverfahren zu machen, durchaus verständlich. Zumal an immer mehr Orten im Wissenschaftssystem festgestellt wird, dass Peer Review und Rankings an ihre Grenzen stoßen. So konstatiert Helga Nowotny (2018), Wissenschaftsforscherin und Gründungsmitglied des European Research Council, dass „das Peer-Review-System weltweit am Rand des Kollapses (steht), da es die Fülle an Publikationen nicht mehr angemessen verarbeiten kann. [...] Wir sollten daher darauf dringen, die Anzahl wissenschaftlicher Publikationen in den nächsten zehn Jahren zu halbieren, um die Qualität entsprechend zu steigern. Doch da müssen alle mitmachen."

Dass da alle mitmachen, ist fraglich. Längst ist der „Tsunami des New Public Management über die Universitäten hinweggefegt und hat sie und ihre Wissenschaftler in einen Verdrängungswettbewerb gerissen" (Kieser 2016, S. 468). In solchen Zeiten nun - und auf den ersten Blick wie um dieser Entwicklung
Prof. Dr. Klaus-

Dieter Altmeppen

lehrt Journalistik

an der Katholischen

Universität Eichstätt-

Ingolstadt und ist

Mitherausgeber von

Communicatio

Socialis.

Prof. Dr. Alexander

Filipović ist Inhaber

der Professur für

Medienethik an

der Hochschule

für Philosophie

in München und

Mitherausgeber von

Communicatio

Socialis. 
zu trotzen - annonciert Communicatio Socialis eine Entscheidung gegen den Trend: Die Einführung eines (modifizierten) Peer-Review-Verfahrens. Das ist begründungspflichtig.

Communicatio Socialis ist die einzige medienethische Zeitschrift im deutschsprachigen Raum. Ihre konzeptionellen Grundlagen kann sie vor allem deshalb erfüllen, weil die Redaktion kontinuierlich Themen sucht, anregt, Beiträger_innen gewinnt und die eingereichten Manuskripte in akribischer Manier bearbeitet, inhaltlich wie formal. Zum Kon-

Communciatio Socialis

fügt sich - zumindest in Teilen dem Push der internationalen

Publikationskultur. zept gehört auch, dass Themenhefte bis zu einem Jahr im Voraus geplant werden (ohne ein Gastherausgeberprinzip). Dabei wird von der Redaktion nicht nur auf die Einreichung von Beiträgen gewartet, sondern kompetente Autor_innen zu bestimmten Themen um ihre Beiträge gebeten. Diese Kontinuität besteht seit vielen Jahren und sie ist Verpflichtung für Communicatio Socialis.

Ein Reviewingprozess mit externen Gutachter_innen passt nicht in diese Ablauforganisation, und er ist auch nicht nötig angesichts der umfassenden redaktionellen Betreuung der $\mathrm{Au}-$ tor_innen und Bearbeitung der Beiträge. Ein intensives Reviewing durch eine thematisch hoch kompetente Redaktion ist schon lange üblich bei Communicatio Socialis. Zudem sind Kriterien eines Peer Review-Verfahrens aufgrund der Konzeption und des Planungsprozesses der Hefte nur schwerlich umzusetzen.

Warum führt Communciatio Socialis dann trotzdem ein Peer Review-Verfahren ein? Aufgrund mehrfacher Nachfragen und vieler Gespräche mit Kolleg_innen können wir davon ausgehen, dass Beiträge, die gern bei Communicatio Socialis eingereicht worden wären, doch an andere Zeitschriften geschickt wurden, weil das Peer Review-Verfahren fehlt. Reviewte Beiträge benötigen offensichtlich diejenigen, die auf Publikationsmöglichkeiten für wissenschaftliche Beiträge über Medien-, Digital- und Informationsethik, über Normen, Moral und Werte und/oder über Sollenserwartungen generell wie in der öffentlichen Kommunikation angewiesen sind, zum Beispiel bei kumulativen Promotionsverfahren oder für Berufungsverfahren.

Communciatio Socialis fügt sich - zumindest in Teilen - dem Push der internationalen Publikationskultur. Die Entwicklung der Publikationsstrukturen fordern ein entsprechendes Handeln von Communicatio Socalis. Herausgeber und Redaktion sind sich des schmalen Grats bewusst, auf dem sie wandeln. Den Tsunami der peer-reviewten Artikel ethisch wie fachpoli- 
tisch zu kritisieren und gleichzeitig selbst Teil dieses funktional geregelten Wissenschaftssystems anzubieten, ist widersprüchlich. Aus diesem Grund führt Communicatio Socialis ein modifiziertes Reviewverfahren ein. Auch weiterhin werden alle Artikel zur Qualitätssicherung ein internes Begutachtungsverfahren in der Redaktion durchlaufen. Zusätzlich bietet Communication Socialis Autor_innen auf deren ausdrücklichen Wunsch ein Peer Review-Verfahren mit externen Gutachter_innen an. Peer review by request könnte dieses Verfahren ,neudeutsch“ genannt werden. Nähere Informationen zu diesem Vorgehen finden Sie im Autor_innenmerkblatt. Extern begutachtete Artikel werden künftig in der Fußzeile durch den Hinweis 'Extern begutachteter Artikel (Peer-Reviewed)' entsprechend gekennzeichnet.

Communicatio Socialis geht somit einen dritten Weg zwischen Redaktions- und Peer Review. Und Communicatio Socialis beteiligt die Autor_innen an dieser Entscheidung. Denn die können künftig entscheiden, ob sie ein Peer Review-Verfahren für ihren Beitrag wünschen. Das Review der Redaktion bleibt ihnen damit nicht erspart, denn an diesem Qualitätsmerkmal hält Communicatio Socialis fest.

\section{Literatur}

Dannenberg, Pascale Anja (2017): Auf der Suche nach der verlorenen Qualität. In: duz Magazin, Nr.6 vom 23. 6., S. 22-27.

Frey, Bruno S./ Osterloh, Margit (2016): Würfeln in der Wissenschaft? Über die Verbindung von Expertenurteilen und Zufall. In: Forschung und Lehre, 23. Jg., H. 2, S. $134 f$.

Guenther, Lars (2017): Kumulative Promotion: Vor- und Nachteile. In: Aviso, Nr. 64, S. $5 f$.

Kieser, Alfred (2016): Ineffizient, irreführend und teuer: wissenschaftliche Zeitschriften in der Krise. Ansätze zur Reform des Systems wissenschaftlicher Veröffentlichungen. In: Die Betriebswirtschaft DBW, 76. Jg., H. 6, S. $467-476$.

Nowotny Helga (2018): Die Ungewissheit umarmen. In: Die Zeit, Nr. 32. 